The Free Internet Journal

for Organic Chemistry
Paper

Arkivoc 2018, part iv, 6-12

\title{
Synthesis of three tricholoma-derived indoles via an ortho-quinone methide
}

\author{
Joshua A. Homer and Jonathan Sperry*
}

School of Chemical Sciences, University of Auckland, 23 Symonds St., Auckland, New Zealand Email: j.sperry@auckland.ac.nz

Dedicated to Professor Gordon Gribble in celebration of his many outstanding contributions to organic synthesis

Received 01-08-2018

Accepted 01-27-2018

Published on line $02-04-2018$

\section{Abstract}

Three Tricholoma-derived indole natural products have been synthesised via an ortho-quinone methide (o$\mathrm{QM})$, itself generated from a phenolic Mannich base.<smiles>COCCN=C1C(=O)C=Cc2[nH]c(C)cc21</smiles><smiles>COCc1c(OC)ccc2[nH]c(C)cc12</smiles><smiles>COc1ccc2[nH]c(C)cc2c1C</smiles><smiles>COc1cc(OC)c2[nH]c(C)cc2c1C</smiles>

Tricholoma-derived indoles

Keywords: Natural product, ortho-quinone methide, indole, $\mathrm{C}-\mathrm{H}$ borylation 


\section{Introduction}

Tricholoma is a large genus of white-spored, gilled mushrooms notable for their distinctive pungency and bitter taste. ${ }^{1,2}$ Several indole alkaloids harbouring a methyl group at the C2-position have been isolated from species of Tricholoma, a selection of which (1-4) can be seen in Figure $1 \mathrm{~A} .{ }^{3-6}$ Unlike most indole alkaloids, the Tricholoma indoles are not biosynthetically derived from tryptophan, but instead assembled via a biosynthetic pathway that involves lascivol (5), a bitter component isolated from Tricholoma lascivum that likely serves as a predation deterrent. ${ }^{7}$ Tellingly, lascivol degrades into the natural product 5-methoxy-2,4-dimethylindole (1) and dimethylglutamate upon treatment with acid in methanol (Figure 1B). ${ }^{7}$ As part of an ongoing synthetic study towards the bisindole natural product sciodole (4), we required a supply of the 'lower half' of this alkaloid, specifically 5-methoxy-2,4-dimethylindole (1). Although 5-methoxy-2,4-dimethylindole (1) has been synthesized previously, ${ }^{8}$ this route starts with a trifluoromethylbenzoquinone that is not readily attainable. Here, we report a synthesis of $\mathbf{1}$ via an ortho-quinone methide (o-QM) that has enabled access to grams of the natural product. Furthermore, the Tricholoma indoles $\mathbf{2}$ and $\mathbf{3}$ were also accessed via the same $o$-QM.

(A)
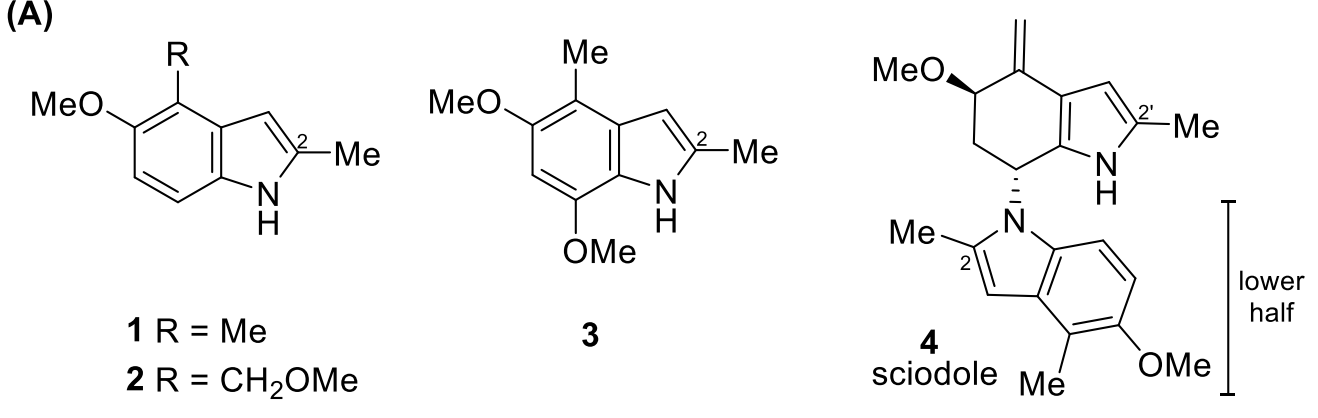

(B)

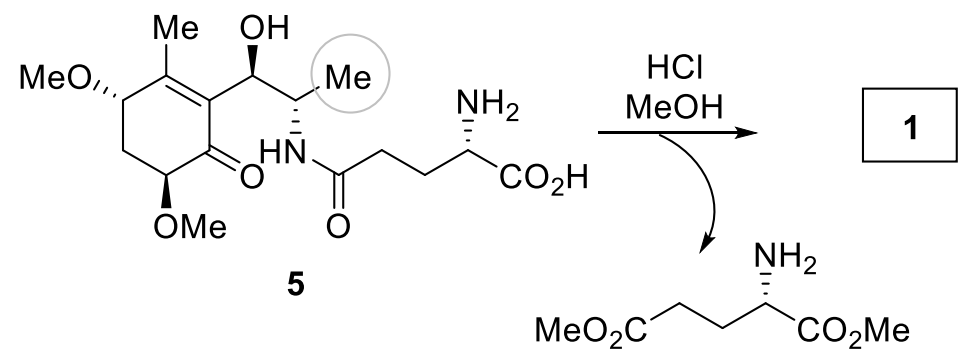

Figure 1. (A) Tricholoma-derived 2-methylindole derivatives (1-4); (B) lascivol (5) and its conversion into 5methoxy-2,4-dimethylindole (1).

\section{Result and Discussion}

The known ${ }^{9}$ 5-hydroxy-2-methylindole 6 was subjected to a regioselective aminomethylation to give the phenolic Mannich base 7,10 which would serve as an 0 -QM precursor. ${ }^{11}$ Upon reaction of the 0 -QM with an appropriate nucleophile, functionalization of the indole C4-site would occur and thus provide access to the targets 1-3 (Scheme 1). Upon treating Mannich base 7 with sodium borohydride in ethanol at reflux, the desired product $\mathbf{8}$ was isolated. In this instance, thermal generation of the o-QM is followed by reduction/aromatization ${ }^{12}$ to give the indole 8 bearing a methyl group at C4. Selective O-methylation of 8 gave the natural product 5-methoxy-2,4-dimethylindole 1, a key intermediate in our ongoing efforts towards the 
synthesis of sciodole (4). Our next target was the natural product 2, which required the introduction of a methoxymethyl group at C4. In this instance, the dimethylamino group of $\mathbf{7}$ was quaternized with iodomethane to allow a base-mediated $o$-QM formation at ambient temperature; ${ }^{13,14}$ trapping of the $o$-QM with methoxide and concomitant phenol alkylation (Mel) occurred to give the natural product 2 in a single-pot from 7. Finally, natural product $\mathbf{3}$ was targeted, which required introduction of a methoxy group at C7 in $\mathbf{1}$. This desired transformation aligns perfectly with the iridium-catalyzed $\mathrm{C}-\mathrm{H}$ borylation reaction, ${ }^{15}$ the regiochemical outcome of which is reliably dictated by the directing ability of the indole $\mathrm{N}-\mathrm{H}$. Subjecting 1 to an iridium-catalyzed $\mathrm{C}-\mathrm{H}$ borylation using the 3,4,7,8-tetramethylphenanthroline as ligand (Me ${ }_{4} \mathrm{Phen}^{16,17}$ gave the 7-borylindole 9 in good yield. A DMAP-assisted ${ }^{18}$ Chan-Evans-Lam coupling of 9 with methanol gave the natural product 5,7-dimethoxy-2,4-dimethylindole (3). The modest yield for this step can be attributed to the instability of the electron-rich indole 3.

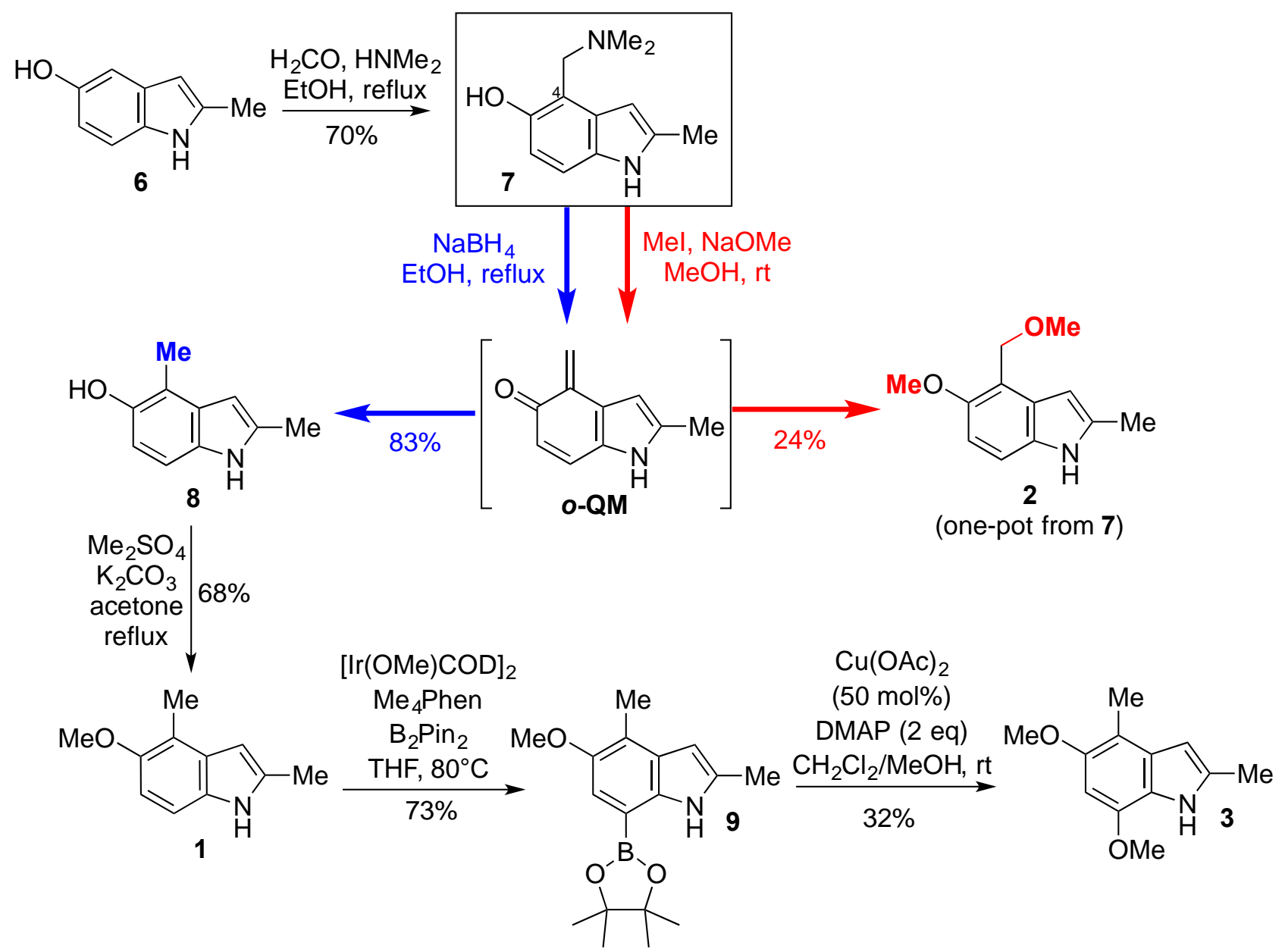

Scheme 1. Synthesis of Tricholoma-derived indoles 1-3 via an o-QM.

\section{Conclusions}

The synthesis of three Tricholoma-derived indole natural products has been achieved. The Mannich base 7 served as a precursor to an o-QM that upon reaction with an appropriate nucleophile, led to efficient installation of a methyl group and a methoxymethyl group at the indole C4-position. The utility of the iridium 
catalyzed $\mathrm{C}-\mathrm{H}$ borylation reaction for selective functionalization of the indole $\mathrm{C} 7$-site has also been demonstrated.

\section{Experimental Section}

General. Commercially available reagents were used throughout without purification unless otherwise stated. Anhydrous solvents were used as supplied. All reactions were routinely carried out in oven-dried glassware under a nitrogen atmosphere unless otherwise stated. Analytical thin layer chromatography was performed using silica plates and compounds were visualized at 254 and/or $360 \mathrm{~nm}$ ultraviolet irradiation followed by staining with either alkaline permanganate or ethanolic vanillin solution. Infrared spectra were obtained using a Perkin Elmer spectrum One Fourier Transform Infrared spectrometer as thin films between sodium chloride plates. Absorption maxima are expressed in wavenumbers $\left(\mathrm{cm}^{-1}\right)$. Melting points were recorded on an Electrothermal melting point apparatus and are uncorrected. NMR spectra were recorded using either a Bruker DRX300 spectrometer operating at $300 \mathrm{MHz}$ for ${ }^{1} \mathrm{H}$ nuclei and $75 \mathrm{MHz}$ for ${ }^{13} \mathrm{C}$ nuclei or a Bruker DRX400 spectrometer operating at $400 \mathrm{MHz}$ for ${ }^{1} \mathrm{H}$ nuclei and $100 \mathrm{MHz}$ for ${ }^{13} \mathrm{C}$ nuclei. Chemical shifts are reported in parts per million (ppm) relative to the tetramethylsilane peak recorded as $\delta 0.00 \mathrm{ppm}$ in $\mathrm{CDCl}_{3} / \mathrm{TMS}$ solvent, or the residual chloroform ( $\delta 7.26 \mathrm{ppm})$ and DMSO ( $\delta 2.50 \mathrm{ppm})$ peaks. The ${ }^{13} \mathrm{C}$ NMR values were referenced to the residual chloroform ( $\delta 77.1 \mathrm{ppm})$ and DMSO ( $\delta 39.5 \mathrm{ppm})$ peaks. ${ }^{13} \mathrm{C}$ NMR values are reported as chemical shift, $\delta$, multiplicity and assignment. ${ }^{1} \mathrm{H}$ NMR shift values are reported as chemical shift, $\delta$, relative integral, multiplicity ( $s$, singlet; $d$, doublet; $t$, triplet; q, quartet; $m$, multiplet), and assignment. Assignments are made with the aid of NOESY and HMBC experiments. High resolution mass spectra were obtained by electrospray ionization in positive ion mode at a nominal accelerating voltage of $70 \mathrm{eV}$ on a Bruker micrOTOFQII mass spectrometer.

5-Hydroxy-4-(dimethylamino)methyl-2-methylindole (7). Prepared according to the procedure reported by Monti and Johnson, ${ }^{10}$ but with changes to purification. The product 7 was not completely characterised in the literature ${ }^{10} \mathrm{~A}$ solution of formaldehyde ( $37 \%$ aqueous, $1.53 \mathrm{~mL}$ ) and dimethylamine ( $40 \%$ aqueous, $2.84 \mathrm{~mL}$ ) in $\mathrm{EtOH}(30 \mathrm{~mL})$ was prepared and warmed to $80^{\circ} \mathrm{C}$ for $30 \mathrm{~min}$. This solution was then cooled to rt before $5-$ hydroxy-2-methylindole $(6)^{9}(3.30 \mathrm{~g}, 22.42 \mathrm{mmol})$ in EtOH $(70 \mathrm{~mL})$ was added. The mixture was stirred under reflux for $1 \mathrm{~h}$, during which time a colour change from yellow to dark red was observed. The reaction mixture was concentrated in vacuo and the resulting crude material was purified by flash column chromatography on silica gel eluting with $\mathrm{CH}_{2} \mathrm{Cl}_{2} \mathrm{MeOH} / \mathrm{NH}_{4} \mathrm{OH}(94: 5: 1)$ to afford the title compound $(3.187 \mathrm{~g}, 15.60 \mathrm{mmol}, 70 \%)$ as a light brown solid, $\mathrm{mp} 128-132{ }^{\circ} \mathrm{C}$ (lit. ${ }^{10} 130-131{ }^{\circ} \mathrm{C}$ ); $v_{\max }$ (neat)/cm ${ }^{-1} 3392,2981,2949,2826,2780,1706$, 1621, 1596, 1555, 1511, 1439, 1427, 1361, 1319, 1270, 1202, 1055, 1039, 1000, 991, 837, 794, 774, 748, 737, 674; $\delta_{\mathrm{H}}\left(400 \mathrm{MHz}, \mathrm{CDCl}_{3}\right) 7.71(1 \mathrm{H}, \mathrm{s}, \mathrm{NH}), 7.07(1 \mathrm{H}, \mathrm{d}, J$ 9.2, $\mathrm{ArH}), 6.69(1 \mathrm{H}, \mathrm{d}, J$ 8.8, ArH), $6.08(1 \mathrm{H}, \mathrm{m}, \mathrm{ArH})$, $3.83\left(2 \mathrm{H}, \mathrm{s}, \mathrm{CH}_{2} \mathrm{NMe}_{2}\right), 2.42(3 \mathrm{H}, \mathrm{s}, \mathrm{Me}), 2.36\left(6 \mathrm{H}, \mathrm{s}, \mathrm{NMe}_{2}\right) ; \delta_{\mathrm{c}}\left(100 \mathrm{MHz}, \mathrm{CDCl}_{3}\right) 151.4$ (C), 135.7 (C), 130.4 (C), $128.4(\mathrm{C}), 111.2(\mathrm{CH}), 110.02(\mathrm{C}), 109.97(\mathrm{CH}), 97.4(\mathrm{CH}), 59.4\left(\mathrm{CH}_{2}\right), 44.8\left(\mathrm{NMe}_{2}\right), 13.8$ (Me); HRMS (ESI) found: $205.1333\left[\mathrm{C}_{12} \mathrm{H}_{16} \mathrm{~N}_{2} \mathrm{O}+\mathrm{H}\right]^{+}$requires 205.1335 .

5-Hydroxy-2,4-dimethylindole (8). To a stirred solution of 7 (2.886 g, $14.07 \mathrm{mmol}$ ) in EtOH (150 mL) cooled to $0{ }^{\circ} \mathrm{C}$, was slowly added $\mathrm{NaBH}_{4}(2.660 \mathrm{~g}, 70.35 \mathrm{mmol})$. The resulting slurry was stirred under reflux for $6 \mathrm{~h}$, after which time the solution was cooled back to $0{ }^{\circ} \mathrm{C}$ and an additional portion of $\mathrm{NaBH}_{4}(1.064 \mathrm{~g}, 28.14 \mathrm{mmol})$ was added. The reaction mixture was stirred under reflux for a further $1 \mathrm{~h}$ before being cooled again to $0{ }^{\circ} \mathrm{C}$ and quenched by the slow addition of $\mathrm{H}_{2} \mathrm{O}(100 \mathrm{~mL})$. The resulting slurry was allowed to gradually warm to $\mathrm{rt}$. The 
aqueous phase was extracted with EtOAc $(5 \times 75 \mathrm{~mL})$. The organic extracts were combined, washed with brine $(100 \mathrm{~mL})$ and dried over $\mathrm{Na}_{2} \mathrm{SO}_{4}$, filtered and concentrated in vacuo. The resulting crude material was purified via flash column chromatography on silica gel eluting with EtOAc/petroleum ether (2:3) to afford the title compound $(1.875 \mathrm{~g}, 11.70 \mathrm{mmol}, 83 \%)$ as an off-white solid, $\mathrm{mp} 65-67^{\circ} \mathrm{C} ; v_{\max }(\mathrm{neat}) / \mathrm{cm}^{-1} 3503,3380,2921$, $1590,1498,1425,1386,1360,1330,1302,1279,1206,1161,1129,1112,1060,1015,897,790,755,739 ; \delta_{H}$ $\left(400 \mathrm{MHz}, \mathrm{CDCl}_{3}\right) 7.72(1 \mathrm{H}, \mathrm{s}, \mathrm{NH}), 7.00(1 \mathrm{H}, \mathrm{d}, \mathrm{J}$ 8.5, $\mathrm{ArH}), 6.66(1 \mathrm{H}, \mathrm{d}, \mathrm{J}$ 8.5, ArH), $6.16(1 \mathrm{H}, \mathrm{s}, \mathrm{ArH}), 4.31(1 \mathrm{H}$, s, OH), $2.43\left(3 \mathrm{H}, \mathrm{d}, J\right.$ 0.8, Me), $2.38(3 \mathrm{H}, \mathrm{s}, \mathrm{Me}) ; \delta_{\mathrm{C}}\left(100 \mathrm{MHz}, \mathrm{CDCl}_{3}\right) 146.8$ (C), 135.7 (C), 131.1 (C), 130.3 (C), $112.7(\mathrm{C}), 110.7(\mathrm{CH}), 108.1(\mathrm{CH}), 99.0(\mathrm{CH}), 14.0(\mathrm{Me}), 11.9(\mathrm{Me})$; HRMS (ESI) found: 184.0735 [C $10 \mathrm{H}_{11} \mathrm{NO}^{+}$ $\mathrm{Na}]^{+}$requires 184.0733 .

5-Methoxy-2,4-dimethylindole (1). To a stirred solution of 8 (1.00 g, $6.20 \mathrm{mmol})$ and $\mathrm{K}_{2} \mathrm{CO}_{3}(2.143 \mathrm{~g}, 15.51$ $\mathrm{mmol})$ in $\mathrm{Me} \mathrm{CO}_{2}(60 \mathrm{~mL})$ was added dimethyl sulfate $(0.880 \mathrm{~mL}, 9.30 \mathrm{mmol})$. The mixture was stirred under reflux for $36 \mathrm{~h}$ after which the reaction was quenched by the addition of aq $\mathrm{NaOH}(1 \mathrm{M}, 10 \mathrm{~mL})$. The resulting aqueous phase was diluted with $\mathrm{H}_{2} \mathrm{O}(20 \mathrm{~mL})$ and extracted with EtOAc $(3 \times 25 \mathrm{~mL})$. The combined organic phases were washed with brine $(20 \mathrm{~mL})$, dried over $\mathrm{Na}_{2} \mathrm{SO}_{4}$, filtered and concentrated in vacuo. The resulting crude material was purified via flash column chromatography on silica gel eluting with EtOAc/petroleum ether (1:4) to afford the title compound (742 mg, $4.24 \mathrm{mmol}, 68 \%$ ) as a colourless solid, $\mathrm{mp} 56-59^{\circ} \mathrm{C}$ (lit. ${ }^{8} 50-52{ }^{\circ} \mathrm{C}$ ); $V_{\max }$ (neat) $/ \mathrm{cm}^{-1} 3394,2920,1593,1497,1424,1324,1260,1228,1168,1097,1007,774,736,666 ; \delta_{H}(400$ $\mathrm{MHz}_{\mathrm{CDCl}}$ ) $7.71(1 \mathrm{H}, \mathrm{s}, \mathrm{NH}), 7.07(1 \mathrm{H}, \mathrm{d}, J$ 8.7, $\mathrm{ArH}), 6.80(1 \mathrm{H}, \mathrm{d}, \mathrm{J}$ 8.7, ArH), $6.18(1 \mathrm{H}, \mathrm{m}, \mathrm{ArH}), 3.84(3 \mathrm{H}, \mathrm{s}$, OMe), $2.43(3 \mathrm{H}, \mathrm{d}, J, \mathrm{Me}), 2.39$ (3 H, s, Me); $\delta_{\mathrm{C}}\left(75 \mathrm{MHz}, \mathrm{CDCl}_{3}\right) 151.4$ (C), 136.0 (C), 131.7 (C), 130.5 (C), 116.8 (C), $108.5(\mathrm{CH}), 107.7(\mathrm{CH}), 99.2(\mathrm{CH}), 58.0$ (OMe), 14.1 (Me), 12.2 (Me); HRMS (ESI) found: 176.1067 $\left[\mathrm{C}_{11} \mathrm{H}_{13} \mathrm{NO}+\mathrm{H}\right]^{+}$requires 176.1070 ; spectroscopic data consistent with isolation report. 3,7

5-Methoxy-4-methoxymethyl-2-methylindole (2). Sodium metal (101 mg, $4.41 \mathrm{mmol}$ ) was added portionwise to $\mathrm{MeOH}(10 \mathrm{~mL})$ at $0{ }^{\circ} \mathrm{C}$. The resulting solution was allowed to stir for $15 \mathrm{~min}$ until the consumption of the sodium was complete. Mannich base $7(300 \mathrm{mg}, 1.47 \mathrm{mmol}$ ) was then slowly added, followed by the dropwise addition of Mel $(0.275 \mathrm{~mL}, 4.41 \mathrm{mmol})$. The resulting solution was allowed to warm to $\mathrm{rt}$ and a nitrogen stream was gently bubbled through the solution for $1 \mathrm{~h}$. After this time, an additional portion of Mel $(0.460$ $\mathrm{mL}, 7.35 \mathrm{mmol}$ ) was added and the resulting reaction was sealed and stirred at $\mathrm{rt}$ for $12 \mathrm{~h}$. The mixture was concentrated in vacuo and the crude material purified by flash column chromatography on silica gel eluting with EtOAc/petroleum ether (2:3) to afford the title compound (73 mg, $0.36 \mathrm{mmol}$, 24\%) as a light brown solid, $\mathrm{mp} 85-88^{\circ} \mathrm{C} ; v_{\max }$ (neat)/cm $\mathrm{cm}^{-1} 3270,2940,1592,1497,1449,1429,1362,1325,1279,1253,1225,1171,1152$, 1097, 1065, 1049, 894, 765, 740; $\delta_{\mathrm{H}}\left(300 \mathrm{MHz}, \mathrm{CDCl}_{3}\right) 7.94(1 \mathrm{H}, \mathrm{br} \mathrm{s}, \mathrm{NH}), 7.14(1 \mathrm{H}, \mathrm{d}, \mathrm{J}$ 8.7, $\operatorname{ArH}), 6.81(1 \mathrm{H}, \mathrm{d}$, J 8.7, $\mathrm{ArH}$ ), $6.32(1 \mathrm{H}, \mathrm{m}, \mathrm{ArH}), 4.82\left(2 \mathrm{H}, \mathrm{d}, J\right.$ 1.5, $\left.\mathrm{CH}_{2}\right), 3.87(3 \mathrm{H}, \mathrm{s}, \mathrm{OMe}), 3.43(3 \mathrm{H}, \mathrm{s}, \mathrm{OMe}), 2.38$ (3 H, s, Me); $\delta_{\mathrm{C}}\left(75 \mathrm{MHz}, \mathrm{CDCl}_{3}\right) 152.1(\mathrm{C}), 136.9$ (C), 131.8 (C), 130.5 (C), 115.7 (C), $110.5(\mathrm{CH}), 107.6(\mathrm{CH}), 99.0(\mathrm{CH}), 66.9$ $\left(\mathrm{CH}_{2}\right), 58.0$ (OMe), 57.8 (OMe), 13.8 (Me); HRMS (ESI) found: $228.0997\left[\mathrm{C}_{12} \mathrm{H}_{15} \mathrm{NO}_{2}+\mathrm{Na}\right]^{+}$requires 228.0995.

The natural product 2 was only detected by mass spectrometry in the isolation report. ${ }^{4}$

5-Methoxy-2,4-dimethyl-7-(4,4,5,5-tetramethyl-1,3,2-dioxaborolan-2-yl)indole (9). [ $\operatorname{lr}(\mathrm{OMe}) \mathrm{COD}]_{2}$ (68 mg, $0.10 \mathrm{mmol}, 6 \mathrm{~mol} \%), 3,4,7,8$-tetramethyl-1,10-phenanthroline $(47 \mathrm{mg}, 0.20 \mathrm{mmol}, 12 \mathrm{~mol} \%$ ) and bis(pinacolato)diboron $(652 \mathrm{mg}, 2.57 \mathrm{mmol})$ in THF $(1.5 \mathrm{~mL})$ was stirred under a heavy stream of nitrogen for approximately $5 \mathrm{~min}$ at $\mathrm{rt}$, during which time the solution became deep green in colour. A solution of 1 (300 $\mathrm{mg}, 1.71 \mathrm{mmol})$ in THF $(1.5 \mathrm{~mL})$ was subsequently added and the resulting mixture sealed under nitrogen and stirred at $80{ }^{\circ} \mathrm{C}$ for $24 \mathrm{~h}$. The mixture was then diluted with EtOAc $(10 \mathrm{~mL})$ and concentrated in vacuo. The resulting crude material was purified via flash column chromatography on silica gel eluting with EtOAc/petroleum ether (1:9) to afford the title compound (376 mg, $1.25 \mathrm{mmol}, 73 \%$ ) as a colourless solid, mp $124-127^{\circ} \mathrm{C} ; v_{\max }\left(\right.$ neat) $/ \mathrm{cm}^{-1} 3445,2976,2928,1607,1561,1503,1445,1388,1370,1292,1211,1167,1137$, 
$1108,971,846,792,757,684 ; \delta_{H}\left(300 \mathrm{MHz}_{\mathrm{CDCl}}\right) 8.69(1 \mathrm{H}, \mathrm{s}, \mathrm{NH}), 7.18(1 \mathrm{H}, \mathrm{s}, \mathrm{ArH}), 6.15(1 \mathrm{H}, \mathrm{m}, \mathrm{ArH}), 3.88$ (3 H, s, OMe), 2.47 (3 H, s, Me), 2.40 (3 H, s, Me), $1.39(12 \mathrm{H}, \mathrm{s}, \mathrm{BPin}) ; \delta_{\mathrm{C}}\left(75 \mathrm{MHz}, \mathrm{CDCl}_{3}\right) 151.1$ (C), 137.2 (C), 135.8 (C), 129.6 (C), 121.2 (C), 113.8 (CH), $98.3(\mathrm{CH}), 83.8$ (2 × C, BPin), 57.7 (OMe), 25.1 (4 × Me, BPin), 14.1 (Me), 12.6 (Me), $1 \times \mathrm{C}$ not observed; HRMS (ESI) found: $324.1732\left[\mathrm{C}_{17} \mathrm{H}_{24} \mathrm{BNO}_{3}+\mathrm{Na}\right]^{+}$requires 324.1744.

5,7-Dimethoxy-2,4-dimethylindole (3). To a solution of 7-borylindole 9 (10 $\mathrm{mg}, 0.033 \mathrm{mmol})$ in a mixture of $\mathrm{CH}_{2} \mathrm{Cl}_{2}(0.75 \mathrm{~mL})$ and $\mathrm{MeOH}(0.25 \mathrm{~mL})$ was added $\mathrm{Cu}(\mathrm{OAc})_{2} \cdot \mathrm{H}_{2} \mathrm{O}(3.3 \mathrm{mg}, 0.016 \mathrm{mmol})$, 4dimethylaminopyridine $(8.0 \mathrm{mg}, 0.066 \mathrm{mmol})$ and molecular sieves $(100 \mathrm{mg})$. The reaction mixture was allowed to stir at rt under an atmosphere of air for $1 \mathrm{~h}$. The mixture was filtered through a plug of Celite and concentrated in vacuo. The resulting crude material was purified via flash column chromatography on silica gel eluting with EtOAc/petroleum ether (1:9) to afford the title compound ( $2.2 \mathrm{mg}, 0.011 \mathrm{mmol}, 32 \%$ yield) as an off white solid; $\mathrm{mp} 172-177^{\circ} \mathrm{C}$ (lit. ${ }^{5} 178-180{ }^{\circ} \mathrm{C}$, dec.); $v_{\max }$ (neat)/cm ${ }^{-1} 3360,2926,2851,1598,1514,1451$, 1398, 1319, 1200, 1125, 1018; $\delta_{\mathrm{H}}\left(300 \mathrm{MHz}_{\mathrm{CDCl}}\right) 7.93(1 \mathrm{H}, \mathrm{br} \mathrm{s}, \mathrm{NH}), 6.38(1 \mathrm{H}, \mathrm{s}, \mathrm{ArH}), 6.16(1 \mathrm{H}, \mathrm{m}, \mathrm{ArH})$, $3.93(3 \mathrm{H}, \mathrm{s}, \mathrm{OMe}), 3.84(3 \mathrm{H}, \mathrm{s}, \mathrm{OMe}), 2.43\left(3 \mathrm{H}, \mathrm{d}, \mathrm{J}\right.$ 0.8, Me), $2.32(3 \mathrm{H}, \mathrm{s}, \mathrm{Me}) ; \delta_{\mathrm{c}}\left(75 \mathrm{MHz}, \mathrm{CDCl}_{3}\right) 151.3(\mathrm{C})$, 143.6 (C), 135.1 (C), 130.7 (C), 121.8 (C), 109.3 (C), 99.5 (CH), $92.4(\mathrm{CH}), 58.8$ (OMe), 55.7 (OMe), 13.9 (Me), 11.6 (Me); HRMS (ESI) found: $228.0997\left[\mathrm{C}_{12} \mathrm{H}_{15} \mathrm{NO}_{2}+\mathrm{Na}\right]^{+}$requires 228.0995; Spectroscopic data consistent with isolation report. ${ }^{5}$

\section{Acknowledgements}

J.S. thanks the Royal Society of New Zealand for a Rutherford Discovery Fellowship. The University of Auckland is thanked for the award of a doctoral scholarship (J.A.H.).

\section{Supplementary Material}

${ }^{1} \mathrm{H} N M R$ and ${ }^{13} \mathrm{C}$ NMR spectra of 1-3 and 7-9.

\section{References}

1. Liu, J. K. Chem. Rev. 2005, 105, 2723.

https://doi.org/10.1021/cr0400818

2. Homer, J. A.; Sperry, J. J. Nat. Prod. 2017, 80, 2178.

https://doi.org/10.1021/acs.jnatprod.7b00390

3. Pang, Z.; Sterner, O. Acta Chem. Scand. 1996, 50, 303.

https://doi.org/10.3891/acta.chem.scand.50-0303

4. Fons, F.; Rapior, S.; Fruchier, A.; Saviuc, P.; Bessiere, J.-M. Cryptogam. Mycol. 2006, 27, 45.

5. Qiu, W.; Kobori, H.; Wu, J.; Choi, J.-H.; Hirai, H.; Kawagishi, H. Biosci. Biotech. Biochem. 2017, 81, 441. https://doi.org/10.1080/09168451.2016.1249453

6. Sterner, O. Nat. Prod. Lett. 1994, 4, 9. https://doi.org/10.1080/10575639408043885

7. Eizenhöfer, T.; Fugmann, B.; Sheldrick, W. S.; Steffan, B.; Steglich, W. Liebigs Ann. Chem. 1990, 1115. https://doi.org/10.1021/jo01269a076 
8. Littell, R.; Allen, G. R. J. Org. Chem. 1968, 33, 2064. https://doi.org/10.1007/s11164-010-0210-X

9. Huang, Y.-S.; Zhang, W.-Q.; Zhang, X.; Wang, J.-Z. Res. Chem. Intermed. 2010, 36, 975. https://doi.org/10.1007/s11164-010-0210-X

10. Monti, S. A.; Johnson, W. O. Tetrahedron 1970, 26, 3685. https://doi.org/10.1016/S0040-4020(01)92947-3

11. Van De Water, R. W.; Pettus, T. R. R. Tetrahedron 2002, 58, 5367. https://doi.org/10.1016/S0040-4020(02)00496-9

12. Gadaginamath, G. S.; Basanagoudar, L. D.; Siddappa, S. J. Indian Chem. Soc. 1977, 54, 709.

13. Geismann, T. A. ; Armen, A. J. Am. Chem. Soc. 1952, 74, 3916 https://doi.org/10.1021/ja01135a061

14. Modica E.; Zanaletti, R.; Freccero, M.; Mella, M. J. Org. Chem. 2001, 66, 41. https://doi.org/10.1021/jo0006627

15. Paul, S.; Chotana, G. A.; Holmes, D.; Reichle, R. C.; Maleczka, R. E. Jr.; Smith, M. R. III. J. Am. Chem. Soc. 2006, 128, 15552.

https://doi.org/10.1021/ja0631652

16. Eastabrook, A. E.; Sperry, J. Aus. J. Chem. 2015, 68, 1810.

17. Eastabrook, A. E.; Sperry, J. Synthesis 2017, 49, 4731. https://doi.org/10.1055/s-0036-1589018

18. Quach T. D.; Batey R. A. Org Lett. 2003, 5, 1381. https://doi.org/10.1021/ol034454n 